\title{
EDITORIAL: MECHANICS IN BIOLOGY
}

Cell and tissue, shell and bone, leaf and flower, are so many portions of matter, and it is in obedience to the laws of physics that their particles have been moved, moulded and confirmed. They are no exception to the rule that God always geometrizes. Their problems of form are in the first instance mathematical problems, their problems of growth are essentially physical problems, and the morphologist is, ipso facto, a student of physical science.

- Sir D’Arcy Wentworth Thompson, On Growth and Form (1917).

The aim of this special issue is to highlight the role of "mechanics in biology" and the important part mathematics has to play in helping us understand biological systems. As D'Arcy Thompson noted over a century ago, biological systems, whether they be cells, tissues, organs or entire bodies, are subject to movement, growth, stresses and strains. Whilst the importance of mechanics in fields like haemodynamics or pulmonary flows has long been appreciated, recently there has been an increasing recognition of how it can drive pattern formation and morphogenesis. This special issue contains papers featuring a wide range of biological topics, including tissue growth (both in vivo and in vitro), swimming microorganisms and fluid flows within the body. We hope that it will go at least some way towards helping readers appreciate the diversity of current research in biological mechanics and the variety of mathematical approaches which are being applied.

Mechanics is, of course, an area of mathematics in which Australia and New Zealand have a long and proud history, traditionally supported by strong bi-directional links with Great Britain. In the case of biological fluid mechanics, an example of the continued fruitfulness of these connections is provided by the career of the late Professor John Blake, who sadly passed away in 2016. As a stalwart of the Australian and UK applied mathematics communities who made seminal contributions to understanding the mechanics of biological systems, a tribute written by his former student and subsequent collaborator David Smith seems a fitting way to open this special issue of the ANZIAM Journal.

The second paper is from another of John Blake's former students, Thomas Montenegro-Johnson, and his collaborator David Gagnon, who provide new insights into the energy expenditure of microorganisms swimming through shear-thinning fluids such as mucus, which are common in biological systems. Their analysis suggests 
that using the common approach of considering a two-dimensional geometry when modelling swimming results in an overestimate of the shear-modulated fluid viscosity, and hence of the energy used in swimming, when compared to the three-dimensional case.

Staying at the microscale, the following paper by Clarke considers the behaviour of a population of light-sensitive swimming microorganisms in a shear flow. These organisms' light sensitivity could potentially be exploited in technological applications, by using light to direct and focus cells into particular areas. However, these applications will usually involve the cells being suspended in a flowing fluid. The paper thus investigates the interaction of the cells' photosensitive response with a background flow and how this affects the patterns observed. The final paper focusing on a cell-scale problem is by Copos and Guy, who consider a single cell within a microfluidic channel, incorporating the effect of the cytoskeleton. They present a new model, treating the cell as a porous, viscoelastic material in a Stokes flow which simulated using the immersed boundary method.

Moving up to the tissue scale, Holden et al. present a multiphase model for tissue growth within a porous scaffold in vitro. Engineering approaches are increasingly being used to culture cells in precisely controlled conditions for applications in regenerative medicine, and mathematical modelling of the processes involved promises, in time, to help grow tissues with the required properties more rapidly. A mathematical highlight of this particular paper is the use of homogenization theory to include the effects of the cells' microenvironment on the macroscopic behaviour of the tissue.

The following two papers are concerned with flows within the body: in branching networks of blood vessels, and in the pulmonary circulation, respectively. The work of Ovenden and Smith is motivated by applications to arteriovenous malformations in the brain, where a vessel branches into many daughters, and which are a significant risk factor for stroke. They consider non-symmetric branchings in three dimensions, using both lattice Boltzmann computations and asymptotic analysis to investigate how the flux through the vessels is affected by the pressure differences and wall shapes. Clark and Tawhai investigate perfusion in the lungs, which is essential to ensure the supply of oxygenated blood to all the tissues of the body. They present an anatomically based model of pulmonary blood flow incorporating gravity, which shows good agreement with data from healthy subjects.

Our last paper, by Erlich et al., considers a problem at the scale of a whole organism - seashell growth. Specifically, they look at organisms where the shell grows from a ring-shaped opening, as in ammonites. Their mathematical model for this process extends previous work by considering the role of mechanical feedback in regulating the growth rate of the mollusc, and how this affects the patterns of ridges or wrinkles observed on the resulting shell. Comparing the predictions of the theory with the fossil record, they find evidence to corroborate the presence of mechanical feedback.

Serendipitously, a seashell appears on the logo of the Society for Mathematical Biology, which (jointly with the Japanese Society for Mathematical Biology) will hold 
its annual conference in Sydney in July 2018. This is the first time this important event for mathematical biologists has been held in Australia, and presents a wonderful opportunity for the Australian and New Zealand mathematical biology communities to showcase their work before an international audience, exchange ideas with colleagues from overseas, renew old friendships and collaborations and, hopefully, forge new ones. We look forward to what will hopefully be a memorable conference!

Finally, we would like to thank all the authors who contributed to this special issue, as well as the expert reviewers who gave their opinions, and the editors and staff at the ANZIAM Journal who have helped us put it together. We hope you enjoy reading it!

ROSEMARY J. DYSON,

School of Mathematics,

University of Birmingham,

Edgbaston,

Birmingham B15 2TT,

UK

email: r.j.dyson@bham.ac.uk

J. EDWARD F. GREEN,

School of Mathematical Sciences,

University of Adelaide,

Adelaide,

South Australia 5005,

Australia

email: edward.green@adelaide.edu.au 